\title{
Avoiding rainbow induced subgraphs in vertex-colorings
}

\author{
Maria Axenovich and Ryan Martin* \\ Department of Mathematics, Iowa State University, Ames, IA 50011 \\ axenovic@iastate.edu, rymartin@iastate.edu
}

Submitted: Jun 25, 2007; Accepted: Jan 4, 2008; Published: Jan 14, 2008

Mathematics Subject Classification: 05C15, 05C55

\begin{abstract}
For a fixed graph $H$ on $k$ vertices, and a graph $G$ on at least $k$ vertices, we write $G \longrightarrow H$ if in any vertex-coloring of $G$ with $k$ colors, there is an induced subgraph isomorphic to $H$ whose vertices have distinct colors. In other words, if $G \longrightarrow H$ then a totally multicolored induced copy of $H$ is unavoidable in any vertex-coloring of $G$ with $k$ colors. In this paper, we show that, with a few notable exceptions, for any graph $H$ on $k$ vertices and for any graph $G$ which is not isomorphic to $H, G \nrightarrow H$. We explicitly describe all exceptional cases. This determines the induced vertexanti-Ramsey number for all graphs and shows that totally multicolored induced subgraphs are, in most cases, easily avoidable.
\end{abstract}

\section{Introduction}

Let $G=(V, E)$ be a graph. Let $c: V(G) \rightarrow[k]$ be a vertex-coloring of $G$. We say that $G$ is monochromatic under $c$ if all vertices have the same color and we say that $G$ is rainbow or totally multicolored under $c$ if all vertices of $G$ have distinct colors. The existence of a graph forcing an induced monochromatic subgraph isomorphic to $H$ is well known. The following bounds are due to Brown and Rödl:

Theorem 1 (Vertex-Induced Graph Ramsey Theorem [6]) For all graphs H, and all positive integers $t$ there exists a graph $R_{t}(H)$ such that if the vertices of $R_{t}(H)$ are colored with $t$ colors, then there is an induced subgraph of $R_{t}(H)$ isomorphic to $H$ which is monochromatic. Let the order of $R_{t}(H)$ with smallest number of vertices be $r_{\text {mono }}(t, H)$. Then there are constants $C_{1}=C_{1}(t), C_{2}=C_{2}(t)$ such that $C_{1} k^{2} \leq \max \left\{r_{\text {mono }}(t, H)\right.$ : $|V(H)|=k\} \leq C_{2} k^{2} \log _{2} k$.

* Research supported in part by NSA grant H98230-05-1-0257 
Theorem 1 is one of numerous vertex-Ramsey results investigating the existence of induced monochromatic subgraphs, including the studies of Folkman numbers such as in [16], [4] and others. There are also "canonical"-type theorems claiming the existence of monochromatic or rainbow substructures (see, for example, a general survey paper by Deuber [13]). The paper of Eaton and Rödl provides the following specific result for vertex-colorings of graphs.

Theorem 2 (Vertex-Induced Canonical Graph Ramsey Theorem [14]) For all graphs $H$, there is a graph $R_{\text {can }}(H)$ such that if $R_{\text {can }}(H)$ is vertex-colored then there is an induced subgraph of $R_{\text {can }}(H)$ isomorphic to $H$ which is either monochromatic or rainbow. Let the order of such a graph with the smallest number of vertices be $r_{c a n}(H)$. There are constants $c_{1}, c_{2}$ such that $c_{1} k^{3} \leq \max \left\{r_{\text {can }}(H):|V(H)|=k\right\} \leq c_{2} k^{4} \log k$.

In this paper, we study the existence of totally multicolored induced subgraphs isomorphic to a fixed graph $H$, in any coloring of a graph $G$ using exactly $k=|V(H)|$ colors. We call a coloring of vertices, with $k$ nonempty color classes, a $k$-coloring. Whereas the induced-vertex Ramsey theory minimizes the order of a graph that forces a desired induced monochromatic graph, it is clear that for the multicolored case a similar goal is trivially achieved by the graph $H$ itself. What is not clear is whether it is possible to construct an arbitrarily large graph $G$ with the property that any $k$-coloring of $V(G)$ induces a rainbow $H$.

Definition 1 Let $G$ and $H$ be two graphs. We say " $G$ arrows $H$ " and write $G \longrightarrow H$ if for any coloring of the vertices of $G$ with exactly $|V(H)|$ colors, there is an induced rainbow subgraph isomorphic to $H$. Let

$$
f(H)=\max \{|V(G)|: G \longrightarrow H\},
$$

if such a max exists. If not, we write $f(H)=\infty$.

It follows from the definition that if $f(H)=\infty$ then for any $n_{0} \in \mathbb{N}$ there is $n>n_{0}$ and a graph $G$ on $n$ vertices such that any $k$-coloring of vertices of $G$ produces a rainbow induced copy of $H$. The function $f$ was first investigated by the first author in [2].

Theorem 3 ([2]) Let $H$ be a graph on $k$ vertices. If $H$ or its complement is (1) a complete graph, (2) a star or (3) a disjoint union of two adjacent edges and an isolated vertex, then $f(H)=\infty$; otherwise $f(H) \leq 4 k-2$.

We improve the bound on $f(H)$ to the best possible bound on graphs $H$ for which $f(H)<\infty$.

Theorem 4 Let $H$ be a graph on $k$ vertices. If $H$ or its complement is (1) a complete graph, (2) a star or (3) a disjoint union of two adjacent edges and an isolated vertex, then $f(H)=\infty$; otherwise $f(H) \leq k+2$ if $k$ is even and $f(H) \leq k+1$ if $k$ is odd.

What we prove in this paper is stronger. First, we find $f(H)$ for all graphs $H$. Second, we are able to explicitly classify almost all pairs $(G, H)$ for which $G \longrightarrow H$. We describe some classes of graphs and state our main result in the following section. 


\section{Main Result}

Let $K_{n}, E_{n}, S_{n}, C_{n}, P_{n}$ be a complete graph, an empty graph, a star, a cycle and a path on $n$ vertices, respectively. Let $H_{1}+H_{2}$ denote the vertex-disjoint union of graphs $H_{1}$ and $H_{2}$. We denote $\Lambda=P_{3}+K_{1}$. If $H$ is a graph, let $\bar{H}$ denote its complement. Let $P$ and $\Theta$ be the Petersen and Hoffman-Singleton graphs, respectively; see Wolfram Mathworld ([17] and [18], respectively) for beautiful pictures.

Let $k H$ denote the vertex-disjoint union of $k$ copies of graph $H$. We write $H \approx H^{\prime}$ if $H$ is isomorphic to $H^{\prime}$ and we say that $H \in\left\{H_{1}, H_{2}, \ldots\right\}$ if there exists an integer $i$ for which $H \approx H_{i}$. We write $G-v$ to denote the subgraph of $G$ induced by the vertex set $V(G) \backslash\{v\}$. A graph is vertex-transitive if, for every distinct $v_{1}, v_{2} \in V(G)$, there is an automorphism, $\varphi$, of $G$ such that $\varphi\left(v_{1}\right)=v_{2}$. A graph is edge-transitive if, for every distinct $\left\{x_{1}, y_{1}\right\},\left\{x_{2}, y_{2}\right\} \in E(G)$, there is an automorphism, $\varphi$, of $G$ such that either both $\varphi\left(x_{1}\right)=x_{2}$ and $\varphi\left(y_{1}\right)=y_{2}$ or both $\varphi\left(x_{1}\right)=y_{2}$ and $\varphi\left(y_{1}\right)=x_{2}$.

Let $P^{\prime}$ and $\Theta^{\prime}$ be the graphs obtained by deleting two nonadjacent vertices from $P$ and $\Theta$, respectively. In the proof of Lemma 7, we establish that both $\bar{P}$ and $\bar{\Theta}$ are edgetransitive, thus $P^{\prime}$ and $\Theta^{\prime}$ are well-defined. For $\ell \geq 3$, let $M_{\ell}$ denote a matching with $\ell$ edges; let $M_{\ell}^{\prime}$ denote the graph obtained by deleting two nonadjacent vertices from $M_{\ell}$. We say that a graph is trivial if it is either complete or empty.

We define several classes of graphs in order to prove the main theorem.

Let $\mathcal{C}$ denote the class of connected graphs on at least three vertices.

Let $\mathcal{P}_{3}^{\prime}$ denote the set of graphs $G=(V, E)$ such that there is a nontrivial vertexpartition $V=V_{1} \cup V_{2} \cup V_{3}$, with (a) $V_{i} \neq \emptyset$, for all $i=1,2,3$, (b) the tripartite subgraph of $G$ obtained by deleting all edges with both endpoints in $V_{i}, i=1,2,3$ is a vertex disjoint union of complete tripartite graphs and bipartite graphs, each with vertices in only two of the parts $V_{1}, V_{2}, V_{3}$; see Figure 1 . Let $\mathcal{P}_{3}$ be the set of all graphs on at least 4 vertices which are not in $\mathcal{P}_{3}^{\prime}$.

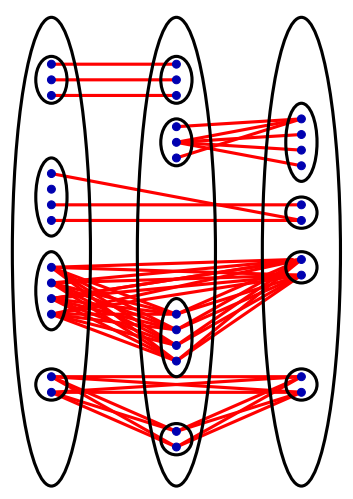

Figure 1: A graph from class $\mathcal{P}_{3}^{\prime}$.

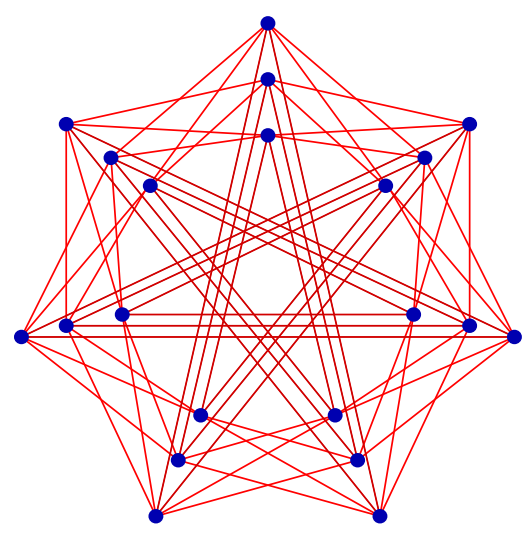

Figure 2: Graph $G(3)$ which arrows $\Lambda$.

Let $\mathcal{L}=\{G(m): m \geq 1\}$, where $G(m)=(V, E), V=\{v(i, j): 0 \leq i \leq 6,1 \leq j \leq m\}$, $E=\{v(i, j) v(i+1, k): 1 \leq j, k \leq m, j \neq k, 0 \leq i \leq 6\} \cup\{v(i, j) v(i+3, j): 1 \leq j \leq$ 
$m, 0 \leq i \leq 6\}$, addition is taken modulo 7 , see Figure 2 for an illustration.

Let $\mathcal{T}$ denote the set of graphs $T$ such that (a) neither $T$ nor $\bar{T}$ is complete or a star, and (b) either $T$ is vertex-transitive or there exists a vertex, $v$ of degree 0 or $|V(T)|-1$ such that $T-v$ is vertex-transitive. Note that a perfect matching is an example of a graph in $\mathcal{T}$. If $T \in \mathcal{T}$, denote $T^{\prime}$ to be the graph that is obtained from $T$ by deleting a vertex $w$ that is neither of degree 0 nor of degree $|V(T)|-1$. Let $\mathcal{T}^{\prime}=\left\{T^{\prime}: T \in \mathcal{T}\right\}$. Note that, given $T^{\prime} \in \mathcal{T}^{\prime}$, the corresponding graph $T \in \mathcal{T}$ is unique.

Let $\mathcal{F}_{\infty}=\left\{K_{k}, \overline{K_{k}}: k \geq 2\right\} \cup\left\{S_{k}, \overline{S_{k}}: k \geq 3\right\} \cup\{\Lambda, \bar{\Lambda}\}$. As we see in Theorem 3, $H \in \mathcal{F}_{\infty}$ iff $f(H)=\infty$. Observe (see also [2]) that $G \longrightarrow H$ if and only if $\bar{G} \longrightarrow \bar{H}$. In order to classify all graphs $G$ which arrow $H$, we introduce the following notation

$$
\mathcal{A} \operatorname{rrow}(H)=\{G: G \longrightarrow H, G \not \approx H\} .
$$

\section{Theorem 5 (Main Theorem)}

- $\operatorname{Arrow}(\Lambda) \supseteq \mathcal{L}$,

$$
\begin{aligned}
& \mathcal{A} \operatorname{rrow}\left(K_{k}\right)= \begin{cases}\mathcal{C} & \text { if } k=2, \\
\left\{K_{n}: n>k\right\} & \text { if } k \geq 3,\end{cases} \\
& \mathcal{A} \operatorname{rrow}\left(S_{k}\right)= \begin{cases}\mathcal{P}_{3} & \text { if } k=3, \\
\left\{S_{n}: n>k\right\} & \text { if } k \geq 4,\end{cases}
\end{aligned}
$$

- $\operatorname{Arrow}\left(P^{\prime}\right)=\{P\}, \quad \mathcal{A r r o w}\left(\Theta^{\prime}\right)=\{\Theta\}$, $\mathcal{A} \operatorname{rrow}\left(M_{\ell}^{\prime}\right)=\left\{M_{\ell}, M_{\ell-1}+K_{1}\right\}, \ell \geq 3$,

- $\operatorname{Arrow}\left(T^{\prime}\right)=\{T\}$, if $T^{\prime} \in \mathcal{T}^{\prime}$ and $T^{\prime} \not M_{\ell}^{\prime}, \ell \geq 3$,

- If $H, \bar{H} \notin \mathcal{F}_{\infty} \cup\left\{P^{\prime}, \Theta^{\prime}\right\} \cup \mathcal{T}^{\prime}$, then $\operatorname{Arrow}(H)=\emptyset$.

Corollary 6 Let $H$ be a graph on $k$ vertices. Then

$$
f(H)= \begin{cases}\infty, & H \in \mathcal{F}_{\infty}, \\ k+2, & H \in\left\{P^{\prime}, \Theta^{\prime}, \overline{P^{\prime}}, \overline{\Theta^{\prime}}\right\} \cup\left\{M_{\ell}^{\prime}, \overline{M_{\ell}^{\prime}}: \ell \geq 3, k=2 \ell-2\right\}, \\ k+1, & H \in\left\{T^{\prime}: T^{\prime} \in \mathcal{T}^{\prime}\right\} \backslash\left\{M_{\ell}^{\prime}, \overline{M_{\ell}^{\prime}}: \ell \geq 3, k=2 \ell-2\right\}, \\ k, & \text { otherwise. }\end{cases}
$$

Remark 1 We wish to observe that a graph $H$ for which $f(H)=k+2$ only occurs for even values of $k, k \geq 4$ and is, up to complementation, uniquely defined by $k$ except in the cases of $k=8$ and $k=48$. If $k \in\{8,48\}$, then there are two such complementary pairs of graphs $H$. We also note that $\mathcal{A}$ rrow $(H)$ is fully classified for every graph $H$ except for $H \in\{\Lambda, \bar{\Lambda}\}$. 
This paper is structured as follows: In Section 3 we state without proofs all of the lemmas and supplementary results. In Section 4, we prove the main theorem. In Section 5 we prove all the lemmas from Section 3.

The main technical tool of the proof is the fact that in most cases we can assume that the degree sequence of the graph $H$ is consecutive. Using this, it is possible to show that $f(H) \leq|V(H)|+c$ for some absolute constant $c$ and for all $H$ such that $f(H)<\infty$. We prove several additional cited lemmas which provide a delicate analysis allowing one to get an exact result for ALL graphs, in particular for ones with small maximum degree.

\section{Definitions, Lemmas and supplementary results}

Let $G$ be a graph on $n$ vertices and $v \in V(G)$. The degree of $v$ is denoted $\operatorname{deg}(v)$ and the codegree of $v, n-1-\operatorname{deg}(v)$, is denoted $\operatorname{codeg}(v)$. When the choice of a graph is ambiguous, we shall denote the degree of a vertex $v$ in graph $G$ by $\operatorname{deg}(G, v)$. If vertices $u$ and $v$ are adjacent, we write $u \sim v$, otherwise we write $u \not v$. For subsets of vertices $X$ and $Y$, we write $X \sim Y$ if $x \sim y$ for all $x \in X, y \in Y$; we write $X \not Y$ if $x \neq y$ for all $x \in X, y \in Y$. For a vertex $x \notin Y$, we write $x \sim Y$ if $\{x\} \sim Y$ and $x \not Y$ if $\{x\} \not Y$. For a subset $S$ of vertices of a graph $G$, let $G[S]$ be the subgraph induced by $S$ in $G$. The neighborhood of a vertex $v$ is denoted $N(v)$, and the closed neighborhood of $v, N[v]=N(v) \cup\{v\}$. We shall write $e(G)$ to denote the number of edges in a graph $G$. The subset of vertices of degree $i$ in a graph $G$ is $G_{i}$. The minimum and maximum degrees of a graph $G$ are denoted by $\delta(G)$ and $\Delta(G)$, respectively. For all other standard definitions and notations, see [19].

We say the degree sequence of a graph $H$ is consecutive if, for every $i \in$ $\{\delta(H), \ldots, \Delta(H)\}$, there exists a $v \in V(H)$ such that $\operatorname{deg}(v)=i$. The following definition is important and used throughout the paper.

Definition 2 For a graph $H$ on $k$ vertices, let the deck of $H$, denoted $\operatorname{deck}(H)$, be the set of all induced subgraphs of $H$ on $k-1$ vertices. We say that a graph $F$ is in the deck of $H$ if it is isomorphic to a graph from the deck of $H$. The graph $G$ on $n$ vertices is said to be bounded by a graph $H$ on $k$ vertices if both $\Delta(G)=\Delta(H)$ and $\delta(G)=n-k+\delta(H)$.

For $S \subseteq V(G)$, if $G[S] \approx H$, we say (to avoid lengthy notation), that $S$ induces $H$ in $G$ and we shall label the vertices in $S$ as the corresponding vertices of $H$.

We use the following characterization of regular graphs of diameter 2 .

Theorem 7 (Hoffman-Singleton, [12]) If $G$ is a diameter 2, girth 5 graph which is $\Delta$-regular, then $\Delta \in\{2,3,7,57\}$. Moreover, if $\Delta=2, G$ is the 5 -cycle; if $\Delta=3$, then $G$ is the Petersen graph; and if $\Delta=7, G$ is the Hoffman-Singleton graph. It is not known if such a graph exists for $\Delta=57$. 
Note that if a 57-regular graph of diameter 2 exists, it is called a $(57,2)$-Moore graph.

One of our tools is the following theorem of Akiyama, Exoo and Harary [1], later strengthened by Bosák [7].

Theorem 8 (Bosák's theorem) Let $G$ be a graph on $n$ vertices such that all induced subgraphs of $G$ on $t$ vertices have the same size. If $2 \leq t \leq n-2$ then $G$ is either a complete graph or an empty graph.

In all of the lemmas below we assume that

$$
|V(G)|=n, \quad|V(H)|=k, \quad \Delta=\Delta(H), \text { and } \delta=\delta(H) .
$$

Lemma 1 If $G \longrightarrow H$, then the following holds:

(1) If $\Delta \leq k-3$, then $\Delta(G)=\Delta$.

(2) If $2 \leq \delta \leq \Delta \leq k-3$, then $n \leq k+\Delta-\delta$ with equality iff $\Delta(G)=\delta(G)$.

Lemma 2 If $H$ is a graph on $k \geq 3$ vertices and $G$ is a graph on $n \geq k+2$ vertices such that $G \longrightarrow H$, then either $H$ or its complement is a star or the degree sequence of $H$ is consecutive.

The Deck Lemma is an important auxiliary lemma that is used throughout this paper.

Lemma 3 (Deck lemma) Let $G \longrightarrow H$. For any set $U \subset V(G)$ with $|U|=k-1, G[U]$ is in the deck of $H$. Consequently, $e(H)-\Delta \leq e(G[U]) \leq e(H)-\delta$.

Lemma 4 If $f(H)>k$ and $H$ has consecutive degrees, then $\Delta \leq \delta+3$.

Observe that Lemmas 1,2 and 4 immediately imply that $f(H) \leq|V(H)|+3$ if $2 \leq \delta \leq \Delta \leq k-3$. The remaining lemmas allow us to deal with the cases where $\delta<2$ or $\Delta>k-3$ and to prove exact results.

Lemmas 5 and 6 address the cases where $f(H)=\infty$ and $f(H)=k+1$.

Lemma $5 \operatorname{Arrow}\left(K_{k}\right)= \begin{cases}\mathcal{C}, & k=2, \\ \left\{K_{n}: n>k\right\}, & k \geq 3\end{cases}$

and $\operatorname{Arrow}\left(S_{k}\right)= \begin{cases}\mathcal{P}_{3}, & k=3 \\ \left\{S_{n}: n>k\right\}, & k \geq 4\end{cases}$

Lemma $6\{(G, H): G \longrightarrow H,|V(G)|=k+1\}=\left\{\left(T, T^{\prime}\right): T \in \mathcal{T}\right\}$.

Lemmas 7 and 8 allow us to deal with the case where $n \geq k+2$ and $G$ is regular or almost regular. 
Lemma 7 Assume that $k \geq 3$. Let $Q$ be the set of pairs $(G, H)$ such that $|V(G)| \geq k+2$, $G \longrightarrow H, G$ is bounded by $H, H \notin \mathcal{F}_{\infty}, H$ has consecutive degrees and $G$ is d-regular for some $d \geq 2$. Then $Q=\left\{\left(P, P^{\prime}\right),\left(\bar{P}, \overline{P^{\prime}}\right),\left(\Theta, \Theta^{\prime}\right),\left(\bar{\Theta}, \overline{\Theta^{\prime}}\right)\right\}$.

Lemma 8 Let $|V(G)|=k+2$ and let $G$ be bounded by $H$. If $\Delta-\delta=3$ and $\Delta(G)-\delta(G)=$ 1 , then $G \nrightarrow H$.

The following is a technical lemma used in the proof of the Main Theorem and Lemma 12.

Lemma 9 If $|V(G)| \geq k+2, G \longrightarrow H, \delta=1$, and $\delta(G)<n-k+\delta$, then $\Delta \leq \delta+2=3$. Furthermore, if equality holds, then $\left|H_{3}\right|=1, H_{3} \sim H_{2}$, and there is an $S \subseteq V(G)$ and $v \in V(G) \backslash S$ such that $G[S] \approx H,|N(v) \cap S|=1$ and $v \nsim H_{3} \cup H_{2}$.

Finally, the following lemmas treat the case when $\Delta=\Delta(H) \in\{1,2,3\}$.

Lemma 10 Let $\Delta=1, H \notin \mathcal{F}_{\infty}$ and $|V(G)| \geq k+2$. Then $G \longrightarrow H$ implies that $k$ is even and $(G, H)=\left(M_{k / 2+1}, M_{k / 2+1}^{\prime}\right)$.

Lemma 11 Let $\Delta=2, H \notin \mathcal{F}_{\infty},|V(G)| \geq k+2$ and $\delta(G)<n-k+\delta$. Then, $G \nrightarrow H$.

Lemma 12 Let $\Delta=3, H \notin \mathcal{F}_{\infty},|V(G)| \geq k+2$ and $\delta(G)<n-k+\delta$. Then, $G \nrightarrow H$.

\section{PROOF of the MAIN THEOREM}

Let $H$ be a graph on $k$ vertices. Recall that $\mathcal{F}_{\infty}=\left\{K_{k}, \overline{K_{k}}: k \geq 2\right\} \cup\left\{S_{k}, \overline{S_{k}}: k \geq 3\right\} \cup$ $\{\Lambda, \bar{\Lambda}\}$. If $H \in \mathcal{F}_{\infty}$, then the theorem follows from Lemma 5 and Theorem 3 .

Let $G \longrightarrow H,|V(G)|>k$ and $H \notin \mathcal{F}_{\infty}$. We shall describe all such graphs $G$ on $n$ vertices.

If $n=k+1$, then Lemma 6 claims that $H \approx T^{\prime} \in \mathcal{T}^{\prime}$ and $G \approx T$. Note that $M_{\ell}^{\prime} \in \mathcal{T}^{\prime}$ for all $\ell \geq 3$. If $T^{\prime}=M_{\ell}^{\prime}$, then $T=M_{\ell-1}+K_{1}$. Therefore we may assume that $n \geq k+2$ and $H \notin \mathcal{F}_{\infty}$. By Lemma 2, the degree sequence of $H$ is consecutive.

CASE 1. $G$ is bounded by $H$.

Recall that $G$ being bounded by $H$ means that $\Delta(G)=\Delta$ and $\delta(G)=n-k+\delta$. By Lemma $4, \Delta \leq \delta+3$. Lemma 1 gives that $n \leq k+3$.

First, suppose $G$ is $\Delta$-regular. If $\Delta \geq 2$, then by Lemma $7, G \in\{P, \bar{P}, \Theta, \bar{\Theta}\}$ and $n=k+2$. If $\Delta \leq 1$, then $G$ is a matching. Lemma 10 covers this case and gives that $H \approx M_{k / 2+1}^{\prime}$. 
Second, suppose $G$ is not regular, then

$$
n-k+\delta=\delta(G)<\Delta(G)=\Delta .
$$

Since $\Delta-\delta \leq 3$, Lemma 1 implies that $n-k<3$. The fact that $n \geq k+2$, implies that $n=k+2$. Applying Lemma 1 again, we see that $\Delta-\delta=3$ and $\delta(G)=(n-k)+\delta=2+(\Delta-3)=\Delta-1$. Thus $\Delta(G)-\delta(G)=1$. By Lemma 8 , $G \nrightarrow H$, a contradiction.

CASE 2. $G$ is not bounded by $H$.

By Lemma 1, if $G \longrightarrow H$ and $G$ is not bounded by $H$, then either $\delta(H) \leq 1$ (in the case where $\delta(G)<n-k+\delta$ ) or $\Delta(H) \geq k-2$ (in the case where $\Delta(G)>\Delta$ ). Using the fact that $G \longrightarrow H$ iff $\bar{G} \longrightarrow \bar{H}$, we will assume, without loss of generality, that $\delta(G)<n-k+\delta$ and $\delta \leq 1$.

Using Lemma 9 (when $\delta=1$ ) and Lemma 4 (when $\delta=0$ ), we have that $\Delta \leq 3$. Since $\Delta \in\{1,2,3\}$, Lemmas $10,11,12$ give that $(G, H)=\left(M_{\ell}, M_{\ell}^{\prime}\right)$.

Summarizing CASES 1 and 2, we see that if $n \geq k+2$ and $H \notin \mathcal{F}_{\infty}$, then $n=k+2$ and $H$ or $\bar{H}$ is in $\left\{M_{k / 2+1}^{\prime}, P^{\prime}, \Theta^{\prime}\right\}$. Lemma 10 and the fact that $M_{\ell}^{\prime} \in \mathcal{T}^{\prime}$ for all $\ell \geq 3$ give that $\mathcal{A r r o w}\left(M_{\ell}^{\prime}\right)=\left\{M_{\ell}, M_{\ell-1}+K_{1}\right\}$. Lemma 7 gives that $\mathcal{A} \operatorname{rrow}\left(P^{\prime}\right)=\{P\}$ and $\operatorname{Arrow}\left(\Theta^{\prime}\right)=\{\Theta\}$.

This concludes the proof of Theorem 5 .

\section{Proofs of Lemmas}

\subsection{Proof of Lemma 1}

(1) Since $G \longrightarrow H, \Delta(G) \geq \Delta$. Let $\Delta \leq k-3$. Suppose there exists a vertex $v \in V(G)$ such that $\operatorname{deg}(v)>\Delta$. Color $N(v)$ with the first $\Delta+1+a$ colors, where $a$ is the largest integer such that both $\Delta+1+a \leq \operatorname{deg}(v)$ and $\Delta+1+a \leq k-1$. Color $v$ with color $k$ and color the rest of the vertices (if such exist) with the remaining colors (or color these vertices with color 1 if no colors remain). Any $S \subseteq V(G)$ that induces a rainbow copy of $H$ has a vertex, namely $v$, of degree greater than $\Delta$, a contradiction.

(2) By Part (1), $\Delta(G)=\Delta$. We have that $\Delta(\bar{H})=k-1-\delta(H) \leq k-3$. Hence, $n-1-\delta(G)=\Delta(\bar{G})=\Delta(\bar{H})=k-1-\delta$. So, $\delta(G)=n-k+\delta$ and $\Delta=\Delta(G) \geq \delta(G)=n-k+\delta$. Thus, $n \leq k+\Delta-\delta$ with equality if and only if $\Delta(G)=\delta(G)$. 


\subsection{Proof of Lemma 2}

Let $H$ have the property that there is an $i, \delta(H)<i<\Delta(H)$ such that there is no $w \in V(H)$ with $\operatorname{deg}(w)=i$. Let $L_{i}(H)=\{v \in V(H): \operatorname{deg}(v)<i\}$, and $U_{i}(H)=\{v \in V(H): \operatorname{deg}(v)>i\}$. Let $L_{i}(G)=\{v \in V(G): \operatorname{deg}(v)<i\}$, and let $U_{i}(G)=\{v \in V(G): \operatorname{deg}(v)>n-k+i\}$. Since $G \longrightarrow H$, we may assume that $H \subseteq G$.

Claim 1. $V(H)=L_{i}(H) \cup U_{i}(H)$ and $V(G)=L_{i}(G) \cup U_{i}(G)$.

The first statement of the claim follows from our assumption on $H$. Assume that there is a vertex $v \in V(G)$ with $i \leq \operatorname{deg}(v) \leq n-k+i$. Color $v$ with one color, $N(v)$ with $i$ other colors and $V(G) \backslash N[v]$ with the remaining $k-i-1$ colors. Any induced rainbow subgraph $H^{\prime}$ of $G$ on $k$ vertices must contain $v$ and exactly $i$ of its neighbors. Thus $H^{\prime}$ can not be isomorphic to $H$; i.e., $G \nrightarrow H$, a contradiction. This proves Claim 1.

Claim 2. $U_{i}(H) \subseteq U_{i}(G)$ and $L_{i}(H) \subseteq L_{i}(G)$.

If there is a vertex $w \in U_{i}(H) \cap L_{i}(G)$, then $\operatorname{deg}(G, w) \leq i-1<i+1 \leq \operatorname{deg}(H, w)$, a contradiction. If there is a vertex $w \in L_{i}(H) \cap U_{i}(G)$, then $\operatorname{deg}(H, w) \leq i-1$, $\operatorname{deg}(G, w) \geq n-k+i+1$. Thus, $\operatorname{codeg}(H, w) \geq k-i$ and $\operatorname{codeg}(G, w) \leq k-i-2$, a contradiction $\operatorname{since} \operatorname{codeg}(G, u) \geq \operatorname{codeg}(H, u)$ for all $u \in V(H)$. This proves Claim 2 .

Assume first that $\left|U_{i}(H)\right|=\left|U_{i}(G)\right|=1$ and consider an arbitrary $(k-1)$-subset $U \subseteq L_{i}(G)$. Color the vertices of $U$ with $k-1$ colors and color the rest of $V(G)$ with the remaining color. The induced copy of $H$ must contain the member of $U_{i}(G)$ and so $U \cup U_{i}(G)$ must induce $H$. We may conclude that all $(k-1)$-subsets of $L_{i}(G)$ are isomorphic. Since $\left|L_{i}(G)\right|=n-1 \geq k+1$, Bosák's theorem implies that $L_{i}(G)$ induces a trivial subgraph. Given that $U \cup U_{i}(G)$ must induce $H$ for any such $U$ and the degree sequence is not consecutive, both $G$ and $H$ must be stars.

Now assume that $\left|U_{i}(G)\right| \geq 2$ and $\left|U_{i}(H)\right|=1$. Color as many vertices of $U_{i}(G)$ with distinct colors as possible (at least two, at most $k-1$ ) and color the rest with the remaining colors. Under this coloring, any rainbow subgraph on $k$ vertices will have at least 2 vertices in $U_{i}(G)$, a contradiction to Claim 2 .

Thus, we may assume that $\left|U_{i}(H)\right| \geq 2$ and a complementary argument implies that $\left|L_{i}(H)\right| \geq 2$. Since $n \geq k+2$, it is the case that either $\left|U_{i}(G)\right|>\left|U_{i}(H)\right|$ or $\left|L_{i}(G)\right|>\left|L_{i}(H)\right|$. Without loss of generality, assume the former. We know that $\left|U_{i}(H)\right|=k-\left|L_{i}(H)\right| \leq k-2$. Color $U_{i}(G)$ with $\left|U_{i}(H)\right|+1 \leq k-1$ colors and $L_{i}(G)$ with the remaining colors. Under this coloring, any rainbow subgraph of $G$ will have more than $\left|U_{i}(H)\right|$ vertices in $U_{i}(G)$, a contradiction to Claim 2. 


\subsection{Proof of Lemma 3}

Consider a $(k-1)$-subset $U \subseteq V(G)$. Color its vertices with $k-1$ distinct colors and color the rest of the vertices with the remaining color. Since there is a rainbow copy of $H$ in this coloring, and its vertices must contain $U, G[U]$ must be in the deck of $H$. Since each $(k-1)$-vertex induced subgraph of $H$ has at least $e(H)-\Delta$ and at most $e(H)-\delta$ edges, the second statement of the lemma follows.

\subsection{An important auxiliary lemma}

Recall that $H_{d}=\{w \in V(H): \operatorname{deg}(H, w)=d\}$.

Lemma 13 Let $H$ be a graph on $k$ vertices with consecutive degrees and let $G$ be a graph on $n \geq k+1$ vertices such that $G \longrightarrow H$. Furthermore, let $S=\left\{y_{1}, y_{2}, \ldots, y_{k}\right\} \subseteq V(G)$

such that $G[S] \approx H$. Let $\operatorname{deg}\left(G[S], y_{1}\right) \leq \operatorname{deg}\left(G[S], y_{2}\right) \leq \cdots \leq \operatorname{deg}\left(G[S], y_{k}\right)$. Each of the following is true:

(1) For any $v \in V(G) \backslash S,\left|N(v) \cap\left(S \backslash\left\{y_{k}, y_{k-1}\right\}\right)\right| \geq \Delta-2$. If equality holds, then $\left|H_{\Delta}\right|=1$ and $H_{\Delta} \sim H_{\Delta-1}$. If $H_{\Delta} \supseteq\left\{y_{k}, y_{k-1}\right\}$ and $y_{k} \nsim y_{k-1}$ then for any $v \in V(G) \backslash S,\left|N(v) \cap\left(S \backslash\left\{y_{k}, y_{k-1}\right\}\right)\right| \geq \Delta$.

(2) For any $v \in V(G) \backslash S,\left|N(v) \cap\left(S \backslash\left\{y_{1}, y_{2}\right\}\right)\right| \leq \delta+1$. If equality holds, then $\left|H_{\delta}\right|=1$ and $H_{\delta} \nsim H_{\delta+1}$. Moreover, if $H_{\delta} \supseteq\left\{y_{1}, y_{2}\right\}$ and $y_{1} \sim y_{2}$ then for any $v \in V(G) \backslash S$, $\left|N(v) \cap\left(S \backslash\left\{y_{k}, y_{k-1}\right\}\right)\right| \leq \delta-1$.

(3) There is a vertex $v \in V(G) \backslash S$ such that either $\{v\} \cup S \backslash\left\{y_{k}\right\}$ induces $H$ or $\{v\} \cup S \backslash\left\{y_{1}\right\}$ induces $H$.

\section{Proof.}

(1) Let $U=\{v\} \cup S \backslash\left\{y_{k}, y_{k-1}\right\}$. Using the Deck Lemma and counting edges incident to $y_{k}$ and $y_{k-1}$, we have $e(H)-\Delta \leq e(G[U]) \leq e(H)-\Delta-(\Delta-1)+1+\left|N(v) \cap\left(S \backslash\left\{y_{k}, y_{k-1}\right\}\right)\right|$. It follows that

$$
\left|N(v) \cap\left(S \backslash\left\{y_{k}, y_{k-1}\right\}\right)\right| \geq \Delta-2 .
$$

If $y_{k} \not y_{k-1}$ and both $y_{k}$ and $y_{k-1}$ are of degree $\Delta$, then $\left|N(v) \cap\left(S \backslash\left\{y_{k}, y_{k-1}\right\}\right)\right| \geq \Delta$.

(2) Let $U=\{v\} \cup S \backslash\left\{y_{1}, y_{2}\right\}$. Then $e(H)-\delta \geq e(U) \geq e(H)-\delta-(\delta+1)+\mid N(v) \cap$ $\left(S \backslash\left\{y_{1}, y_{2}\right\}\right) \mid$. Thus, all the statements in this part hold similarly to part (1).

(3) Rainbow color $S \backslash\left\{y_{1}, y_{k}\right\}$ with colors $\{1, \ldots, k-2\}$, both of the vertices in $\left\{y_{1}, y_{k}\right\}$ with color $k-1$ and $V(G) \backslash S$ with color $k$. Regardless of which vertex of color $k-1$ is chosen, the statement holds. 


\subsection{Proof of Lemma 4}

Let $G \longrightarrow H,|V(G)|>k, S \subseteq V(G)$ and $G[S] \approx H$. Lemma 13 part (3) implies two cases:

CASE 1. There is a $v \in V(G) \backslash S$ so that $S \cup\{v\} \backslash\left\{y_{k}\right\}$ induces $H$.

Consequently, $|N(v) \cap S| \geq \Delta$ and, in particular, $\Delta-2 \leq\left|N(v) \cap\left(S \backslash\left\{y_{1}, y_{2}\right\}\right)\right| \leq$ $\delta+1$. The last inequality follows from Lemma 13 part (2).

CASE 2. There is a $v \in V(G) \backslash S$ so that $S \cup\{v\} \backslash\left\{y_{1}\right\}$ induces $H$.

Consequently, $|N(v) \cap S| \leq \delta+1$ and $\delta+1 \geq\left|N(v) \cap\left(S \backslash\left\{y_{k}, y_{k-1}\right\}\right)\right| \geq \Delta-2$. The last inequality follows from Lemma 13 part (1).

In both cases $\Delta-\delta \leq 3$.

\subsection{Proof of Lemma 5}

If $H=K_{2}$ and $G$ is disconnected, then color the vertices in one component of $G$ with color 1 and all other vertices with color 2. Thus $G \nrightarrow K_{2}$. On the other hand, if $G \nrightarrow K_{2}$, then there is a partition of $V(G)=V_{1} \cup V_{2}$ such that $V_{1} \nsim V_{2}$.

If $H=K_{k}, k \geq 3$ and $G \neq K_{n}, n>k$, then $G \nrightarrow H$ follows from the Deck Lemma since $G$ has two nonadjacent vertices or $n<k$. On the other hand, it is obvious that $K_{n} \longrightarrow K_{k}$, for all $n \geq k$.

Let $H=S_{k}$ for $k \geq 4$. Then by the Deck Lemma, we see that $G$ has no induced subgraph isomorphic to $\overline{P_{3}}$ and no $K_{3}$. Thus $\bar{G}$ has no induced $P_{3}$, and therefore $\bar{G}$ is a vertex disjoint union of cliques, which implies that $G$ is a complete multipartite graph. Since $G$ has no $K_{3}, G$ is a complete bipartite graph. If both parts of $G$ contain at least 2 vertices, color the vertices in these parts with disjoint sets of colors such that each part uses at least two colors. Then any rainbow $k$-subgraph is a complete bipartite graph with at least two vertices in each part, a contradiction. So, we conclude that $G$ has only one vertex in one of the parts, thus $G$ is a star.

Let $H=P_{3}$. It is easy to see that if $G \notin \mathcal{P}_{3}$, then the tri-partition $V_{1}, V_{2}, V_{3}$ of $V(G)$ as in the definition of $\mathcal{P}_{3}^{\prime}$ witnesses that $G \nrightarrow P_{3}$ by coloring $V_{1}, V_{2}, V_{3}$ each with distinct colors. Suppose there is a coloring of $V(G)$ with no rainbow copy of $P_{3}$. Let the color classes be $V_{1}, V_{2}, V_{3}$. Let $G^{\prime}$ be a tripartite subgraph of $G$ with parts $V_{1}, V_{2}, V_{3}$ which is obtained from $G$ by deleting all edges with both endpoints in $V_{i}, i=1,2,3$. Consider a connected component $Q$ of $G^{\prime}$ with vertices in all three parts $V_{1}, V_{2}, V_{3}$. We claim that this component is a complete tripartite graph. To see this, consider the maximal complete 
tripartite subgraph $Q^{\prime}$ of $Q$. It is clear that $Q$ has a path with one vertex in each of $V_{1}, V_{2}, V_{3}$. This path must induce a triangle; so $Q \neq \emptyset$. If $Q^{\prime} \neq Q$, then there is a vertex $v \in V(Q) \backslash V\left(Q^{\prime}\right)$ such that $v$ is adjacent to a vertex in $Q^{\prime}$. Without loss of generality assume that $v \in V_{1}$, then $v$ must be adjacent to all vertices of $Q^{\prime}$ in $V_{2}$ and $V_{3}$. Thus $Q^{\prime} \cup\{v\}$ is a complete tripartite graph larger than $Q^{\prime}$, a contradiction. So, $Q^{\prime}=Q$ and $Q$ is a complete tripartite graph. Therefore, each component of $G^{\prime}$ either has vertices in only two parts or is a complete tripartite graph, so $G \notin \mathcal{P}_{3}$.

\subsection{Proof of Lemma 6}

Let $G \longrightarrow H$ and $n=k+1$. Any coloring of $V(G)$ with $k$ colors assigns the same color to some two vertices. Thus, for any $u, v \in V(G)$, either $G-u$ or $G-v$ is isomorphic to $H$. As an immediate consequence, for at least $n-1$ vertices in $G$, the vertex degrees have the same value $d=e(G)-e(H)$. As a result, there are only three possibilities:

CASE 1. $G-w \approx H$ for all $w \in V(G)$.

In particular, $G-u \approx G-v$ for all $u, v \in V(G)$. Then, $G$ is regular. Since an isomorphism from $G-u$ and $G-v$ can be extended to an automorphism of $G$ mapping $u$ to $v$, we see that $G$ is vertex-transitive.

CASE 2. There is exactly one vertex, $v$, such that $G-v \not H$ and $\operatorname{deg}(G, v)=d^{\prime} \notin$ $\{0, n-1\}$.

As before, we have that for some $d, \operatorname{deg}(G, w)=d$ for all $w \in V(G) \backslash\{v\}$. If $d^{\prime}>d$, then the deletion of a $w \in V(G) \backslash N[v]$ gives exactly one vertex of degree $d^{\prime}$ and the rest of degree $d$ or $d-1$, but the deletion of a neighbor of $v$ does not, a contradiction. Similarly, if $d^{\prime}<d$, then the deletion of a $w \in N(v)$ gives exactly one vertex of degree $d^{\prime}-1$ but the deletion of a nonneighbor of $v$ does not, a contradiction. Thus $d^{\prime}=d$.

Let $w \sim v, w^{\prime} \nsim v$. Let $\varphi$ be an isomorphism from $G-w$ to $G-w^{\prime}$. Then, $\varphi$ maps vertices of degree $d-1$ in $G-w$, which correspond to the neighbors of $w$, to vertices of degree $d-1$ of $G-w^{\prime}$, which correspond to the neighbors of $w^{\prime}$. In particular, $\varphi$ maps $v$ to some vertex $x \neq v$. As before, we can extend $\varphi$ to an automorphism of $G$ by mapping $w$ to $w^{\prime}$. The existence of this automorphism implies that $G-v \approx G-x$, and we can apply CASE 1 .

CASE 3. There is exactly one vertex, $v \in V(G)$ such that $G-v \not \approx H$ and $\operatorname{deg}(G, v) \in\{0, n-1\}$.

Assume without loss of generality that $\operatorname{deg}(G, v)=0$. Then $G-v-u \approx G-v-w$ for all $u, w \in V(G-v)$. As in CASE $1, G-v$ is vertex transitive.

The above implies that if $G \longrightarrow H,|V(G)|=k+1$ then $(G, H)=\left(T, T^{\prime}\right)$ for some $T \in \mathcal{T}$. Now, let $T^{\prime} \in \mathcal{T}^{\prime}$. We need to show that $T \longrightarrow T^{\prime}$. Let $\left|V\left(T^{\prime}\right)\right|=k$. If we color 
the vertices of $T$ with $k$ colors then exactly two vertices, say $u$ and $v$ get the same color and the rest are totally multicolored. So, if $T$ is vertex-transitive, then $T-\{u\} \approx T^{\prime}$ and it is rainbow; if $T$ is a union of a vertex transitive graph and an isolated vertex $w$, then without loss of generality $u \neq w$ and $T-\{u\} \approx T^{\prime}$ and it is rainbow; if $T$ has a vertex of degree $k$, the result follows from the previous case by considering $\bar{T}$.

\subsection{Proof of Lemma 7}

Since $G$ is bounded by $H$,

$$
n-k+\delta=\delta(G)=\Delta(G)=\Delta .
$$

By Lemma $4, \Delta-\delta \leq 3$. Therefore, either $n-k=2$ or $n-k=3$.

CASE 1. $n-k=3$.

In this case, inequality (1) becomes

$$
3+\delta=\delta(G)=\Delta(G)=\Delta \leq \delta+3 .
$$

Thus, all the inequalities are equalities, so $\delta=\Delta-3$. This implies that $k \geq 4$. If $k=4$, then $\Delta=3, \delta=0$, and the fact that the degrees are consecutive implies that $H \approx \bar{\Lambda}$, a contradiction to the assumption that $H \notin \mathcal{F}_{\infty}$. Thus, we can assume that $k \geq 5$.

Let $G[S] \approx H$. Let $y, y^{\prime}, w, w^{\prime}$ be vertices in $S$ with degrees $\Delta-3, \Delta-2, \Delta-1, \Delta$, respectively, in $G[S]$. The fact that $G$ is $\Delta$-regular gives that $y, y^{\prime}, w, w^{\prime}$ are adjacent to 3,2,1,0 vertices, respectively, in $V(G) \backslash S$. Note that $y, y^{\prime}$ and some two vertices in $V(G) \backslash S$ span $C_{4}$ in $G$ and $w, w^{\prime}$ and some two vertices in $V(G) \backslash S$ span $\overline{C_{4}}$ in $G$.

Let $U$ be a set of vertices in $G$ spanning a $C_{4}$. Color all vertices of $U$ with color 1 and rainbow color the remaining vertices with colors $\{2, \ldots, k\}$. Under this coloring, there is a rainbow copy of $H$ containing exactly one vertex of $U$. Thus, the set of vertices outside of this copy of $H$ spans at least 2 edges. As a result, $e(H) \geq e(G)-3 \Delta+2$.

Let $U^{\prime}$ be a set of vertices spanning $\overline{C_{4}}$ in $G$. Color all vertices of $U^{\prime}$ with color 1 and rainbow color the remaining vertices with colors $\{2, \ldots, k\}$. Under this coloring, there is a rainbow copy of $H$ containing exactly one vertex of $U^{\prime}$. Thus, the set of vertices outside of this copy of $H$ spans at most 1 edge. As a result, $e(H) \leq e(G)-3 \Delta+1$, a contradiction to the bound of $e(H) \geq e(G)-3 \Delta+2$, derived above.

CASE 2. $n-k=2$.

In this case, inequality (1) becomes

$$
2+\delta=\delta(G)=\Delta(G)=\Delta
$$

Thus $\delta=\Delta-2$. Let $G[S] \approx H$. 
If $n \leq 5$, then $k \leq 3$ so $H \in \mathcal{F}_{\infty}$. Thus, we may assume that $n \geq 6$. By Ramsey's theorem, either $G$ contains a $K_{3}$ or $G$ contains $\overline{K_{3}}$. Without loss of generality, assume that $G$ contains $\overline{K_{3}}$.

Claim 1. For any copy of $H$ in $G$, the two vertices outside of it are not adjacent and $e(H)=e(G)-2 \Delta$.

By coloring the vertices of some copy of $\overline{K_{3}}$ with the color 1 and rainbow coloring the rest of $V(G)$ with colors $\{2, \ldots, k\}$, it is clear that $e(H)=e(G)-2 \Delta$. If there are two adjacent vertices outside of a copy of $H$ in $G$ then $e(H)=e(G)-2 \Delta+1$, a contradiction.

Claim 2. $G$ has diameter 2 .

If $G$ has diameter greater than 2, then either there are two vertices at a distance 3 in $G$ or $G$ is disconnected. If $G$ is disconnected, then color two vertices in one component with color 1 and two vertices in the other component with color 2 and rainbow color the remaining vertices with colors $\{3, \ldots, k\}$. No matter which vertices are chosen, any rainbow graph on $k$ vertices, under this coloring, has all vertices of degree $\Delta$ or $\Delta-1$.

If $G$ is connected and of diameter at least 3, then let $u$ and $v$ be vertices at distance exactly 3 and $(u, x, y, v)$ be a shortest $u$-v-path. Color $u$ and $y$ with color 1 , color $v$ and $x$ with color 2 , and rainbow color the remaining vertices with colors $\{3, \ldots, k\}$. Under this coloring, a rainbow copy of $H$ must contain $x$ and $y$; otherwise, there is an edge outside of a copy of $H$, contradicting Claim 1 . Therefore, $u$ and $v$ are outside of $H$. But $u$ and $v$ do not have a common neighbor, so $H$ has only vertices of degree $\Delta$ and $\Delta-1$.

This contradicts the fact that $\delta=\Delta-2$.

Claim 3. $G$ has no $K_{3}$ and no $C_{4}$.

Assume there is a triangle in $G$. Coloring its vertices with color 1 and the remaining vertices with colors $\{2, \ldots, k\}$ would contradict Claim 1 . If $G$ has a $C_{4}$, color its independent sets with colors 1 and 2 , respectively, and the remaining vertices with colors $\{3, \ldots, k\}$. Under this coloring, any rainbow $k$-vertex graph has $e(G)-2 \Delta+1$ edges, another contradiction to Claim 1.

Claim 4. For any two nonadjacent vertices $u$ and $v, G-u-v \approx H$.

Color $u, v$ and their common neighbor (which exists by Claim 2 and is unique by Claim 3 ) with color 1 and rainbow color the remaining vertices with colors $\{2, \ldots, k\}$. Claim 1 implies that $G-u-v$ must induce a copy of $H$.

Claim 5. $G$ is vertex-transitive.

Let $v, v^{\prime} \in V(G)$, let $x$ and $y$ be neighbors of $v$ and let $x^{\prime}$ and $y^{\prime}$ be neighbors of $v^{\prime}$. There is an isomorphism $\varphi:(G-x-y) \rightarrow\left(G-x^{\prime}-y^{\prime}\right)$ that sends $v$ to $v^{\prime}$, since $v, v^{\prime}$ are the unique degree $\Delta-2$ vertices in the respective copies of $H$. To show that the map $\varphi$ can be extended to an isomorphism of $G$ itself, we will verify, without loss of generality, that $x^{\prime}$ is adjacent to every vertex of $\varphi(N(x))$ and $y^{\prime}$ is adjacent to every vertex of $\varphi(N(y))$. 
First, note that $y$ and every vertex in $N(x) \backslash\{v\}$ have exactly one common neighbor. This neighbor, however, cannot be $v$ or $x$ because $G$ has no $K_{3}$. Moreover, such neighbors are different for each distinct member of $N(x) \backslash\{v\}$ because otherwise that vertex and two of its neighbors would form a $C_{4}$ with $x$.

Therefore, there is an induced matching between $N(x) \backslash\{v\}$ and $N(y) \backslash\{v\}$.

Let $a \in N(x) \backslash\{v\}$. Without loss of generality, suppose $\varphi(a) \in N\left(x^{\prime}\right)$. Let $b$ be any vertex in $N(y) \backslash\{v\}$. If $b \sim a$, then $\varphi(b) \in N\left(y^{\prime}\right)$ because $G$ has no $K_{3}$. If $b \neq a$, then $a$ and $b$ have a common neighbor in $G-x-y$, so $\varphi(b) \in N\left(y^{\prime}\right)$ because $G$ has no $C_{4}$. Therefore, we can conclude that $\varphi(N(y))=N\left(y^{\prime}\right)$ and symmetrically, $\varphi(N(x))=N\left(x^{\prime}\right)$, extending $\varphi$ to an isomorphism of $G$.

Claim 6. If $G \longrightarrow H, G$ contains no $K_{3}$ and $n \geq 6$, then $G \approx P$ or $G \approx \Theta$.

Since $G$ is regular with diameter 2 and girth 5 , the Hoffman-Singleton theorem (Theorem 7 ) gives that the only possibilities for $G$ are $C_{5}, P, \Theta$ or a $(57,2)$-Moore graph, if it exists. Since $n \geq 6, G \neq C_{5}$. According to an unpublished proof due to Graham Higman, printed in Section 3.7 of Cameron [8], if a $(57,2)$-Moore graph exists, then it cannot be vertex-transitive. So, only $P$ and $\Theta$ remain.

Claim 7. $\bar{P}$ and $\bar{\Theta}$ are edge-transitive.

Using the definition of the Petersen graph as a Kneser graph (see Section 1.6 of Godsil and Royle, [9]), it is easy to see that $\bar{P}$ is edge-transitive.

Now, we shall show that $\bar{\Theta}$ is edge-transitive. The automorphism group of $\Theta$ is of order $50 \times 7$ ! (see Brouwer, Cohen and Neumaier [5] or Hafner [11]) and the stabilizer of a vertex $w$ is $S_{7}$, the symmetric group that permutes the neighbors of $w$. Take any pair of nonadjacent vertices $x$ and $y$. Let $v$ be their common neighbor. Any automorphism which fixes $\{x, y\}$ also fixes $v$. So, the subgroup of automorphisms which fix $\{x, y\}$ is of order at most $2 \times 5$ !. By the orbit-stabilizer theorem (see Section 2.2 of [9]), the orbit of a nonedge $\{x, y\}$ is of size at least $\frac{50 \times 7 !}{2 \times 5 !}=1050$. The number of nonedges in $\Theta$ is 1050 , hence $\bar{\Theta}$ is edge-transitive.

Putting all of the claims together, if the vertices of $G \in\{P, \Theta\}$ are colored with $n-2$ colors, then either one color class is of size 3 or two color classes are each of size 2 . There is a pair of nonadjacent vertices that can be deleted so that each vertex that remains is of a different color. In the first coloring, this is because $G$ has no $K_{3}$; in the second, because $G$ has no $C_{4}$.

Since $\bar{G}$ is edge-transitive, the deletion of any nonadjacent vertices produces a graph isomorphic to $P^{\prime}$ or $\Theta^{\prime}$, respectively. By adding the complementary cases, the Lemma follows. 


\subsection{Proof of Lemma 8}

Let $n=k+2, \Delta-\delta=3, \Delta(G)-\delta(G)=1$. Let $S$ be a set of vertices that induces $H$ and let $\Delta=\operatorname{deg}\left(G[S], y_{k}\right) \geq \cdots \geq \operatorname{deg}\left(G[S], y_{1}\right)=\delta$. By Lemma 13, part (3), there are two possibilities, CASE 1 and CASE 2:

CASE 1. There is a $v_{0} \in V(G) \backslash S$ such that $G\left[\left\{v_{0}\right\} \cup S \backslash\left\{y_{k}\right\}\right] \approx H$.

Let $\left\{v_{1}\right\}=V(G) \backslash\left(S \cup\left\{v_{0}\right\}\right)$. Since $\left|N\left(v_{0}\right) \cap\left(S \backslash\left\{y_{k}\right\}\right)\right|=\Delta$ and $\Delta=\Delta(G)$, it is the case that $v_{1} \not v_{0}$. Color $\left\{v_{0}\right\} \cup S \backslash\left\{y_{1}, y_{2}\right\}$ with colors $\{1, \ldots, k-1\}$ and color $\left\{y_{1}, y_{2}, v_{1}\right\}$ with color $k$. In the rainbow copy of $H, v_{1}$ must be chosen; otherwise the resulting rainbow subgraph has at least $e(H)-(\delta+1)+(\Delta-1)>e(H)$ edges.

So, $U:=V(G) \backslash\left\{y_{1}, y_{2}\right\}$ induces $H$. Count the number of edges in the subgraph induced by $U$ :

$$
\begin{aligned}
e(G[U])=e(H) \geq & e(H)-\left|S \cap N\left(y_{2}\right)\right|-\left|S \cap N\left(y_{1}\right)\right| \\
& +\left(\operatorname{deg}\left(G, v_{0}\right)-2\right)+\left(\operatorname{deg}\left(G, v_{1}\right)-2\right) \\
\geq & e(H)-(\Delta-2)-(\Delta-3)+\operatorname{deg}\left(G, v_{0}\right)+\operatorname{deg}\left(G, v_{1}\right)-4 \\
= & e(H)-2 \Delta+5+\Delta+\operatorname{deg}\left(G, v_{1}\right)-4 \\
= & e(H)-\Delta+1+\operatorname{deg}\left(G, v_{1}\right) .
\end{aligned}
$$

So, $\operatorname{deg}\left(G, v_{1}\right) \leq \Delta-1$ but this must occur with equality because $\delta(G)=\Delta-1$. Since $\operatorname{deg}\left(G, v_{0}\right)=\Delta, \operatorname{deg}\left(G, v_{1}\right)=\Delta-1, v_{0} \nsim v_{1}$, we have that

$$
e(H)=e(G)-2 \Delta+1 .
$$

If there are three vertices of degree $\Delta-1$ in $G$, then color each of them with color $k$ and rainbow color the remaining vertices with colors $\{1, \ldots, k-1\}$. Any rainbow colored graph on $k$ vertices under this coloring would have at least $e(G)-2 \Delta+2$ edges, a contradiction to (4). Thus, there are at most two vertices of degree $\Delta-1$ in $G$.

Because $\operatorname{deg}\left(G, v_{1}\right)=\Delta-1$, equality holds in (3) and, in particular, $\left|S \cap N\left(y_{1}\right)\right|=\Delta-3$. Since $\delta(G)=\Delta-1$, we have that $y_{1} \sim\left\{v_{0}, v_{1}\right\}$ and $\operatorname{deg}\left(G, y_{1}\right)=\Delta-1$. Thus, $v_{1}$ and $y_{1}$ are the only vertices of degree $\Delta-1$ in $G$. The vertex $y_{2}$ has degree $\Delta$ in $G$ but the equality in (3) gives that $y_{1} \not y_{2}$ and $\left|S \cap N\left(y_{2}\right)\right|=\Delta-2$. So, $y_{2} \sim\left\{v_{0}, v_{1}\right\}$. Putting all of this information together, we arrive at the fact that $\left\{v_{0}, y_{2}, v_{1}, y_{1}\right\}$ forms an induced copy of $C_{4}$ with $v_{0} \nsim v_{1}$ and $y_{1} \nsim y_{2}$.

If we rainbow color $S \backslash\left\{y_{2}, y_{1}\right\}$ with colors $\{1, \ldots, k-2\}$ and color the vertices in $\left\{y_{2}, y_{1}\right\}$ with color $k-1$ and $\left\{v_{0}, v_{1}\right\}$ with color $k$, then the only possibility for a rainbow graph with $e(G)-2 \Delta+1$ edges in this coloring is $U^{\prime}:=V(G) \backslash\left\{v_{0}, y_{2}\right\}$. Since $G \longrightarrow H$, the graph induced by $U^{\prime}$ must be isomorphic to $H$. Let $w$ be a vertex of degree $\Delta-3$ in $U^{\prime}$, then $\operatorname{deg}(G, w)=\Delta-1$. This forces $w$ to be either $v_{1}$ or $y_{1}$. However, both $v_{1}$ and $y_{1}$ have exactly one neighbor among $\left\{v_{0}, y_{2}\right\}$, giving that $\operatorname{deg}\left(G\left[U^{\prime}\right], v_{1}\right)=\operatorname{deg}\left(G\left[U^{\prime}\right], y_{1}\right)=\Delta-2$, a contradiction. 
CASE 2. There is a $v_{1} \in V(G) \backslash S$ such that $G\left[\left\{v_{1}\right\} \cup S \backslash\left\{y_{1}\right\}\right] \approx H$.

Consider $\bar{G}$ and $\bar{H}$. We have that $\bar{G} \longrightarrow \bar{H}$ and $\bar{G}$ is bounded by $\bar{H}$. Observe that $y_{1}$ in an induced copy of $H$ in $G$ corresponds to $y_{k}$ in the same set of vertices, which induce a copy of $\bar{H}$ in $\bar{G}$. Thus we have CASE 1 for $\bar{G}$ and $\bar{H}$, resulting in contradiction.

\subsection{Proof of Lemma 9}

Recall that $\delta=1$. Suppose $v$ is a vertex such that $\operatorname{deg}(G, v)=\delta(G)<n-k+\delta$. Then $\operatorname{codeg}(G, v) \geq n-(n-k+\delta)=k-\delta=k-1$. Color $k-1$ non-neighbors of $v$ with distinct colors, color the rest of the graph with the remaining colors. Let $S$ induce a rainbow copy of $H$ in $G$ in this coloring.

Suppose $v \in S$. Since $k-1$ non-neighbors of $v$ must be in $S$, we see that $H$ would contain a vertex (namely, $v$ ) with at least $k-1$ non-neighbors, and thus having degree 0 , a contradiction. As a result, $v \notin S$.

Label the vertices of $S$ so that $\Delta=\operatorname{deg}\left(G[S], y_{k}\right) \geq \cdots \geq \operatorname{deg}\left(G[S], y_{1}\right)=\delta$. Let $U=\{v\} \cup S \backslash\left\{y_{k}, y_{k-1}\right\}$. Using Lemma 3, we have that $e(G[U]) \geq e(H)-\Delta$. On the other hand, we know that $v$ has at least $k-1$ non-neighbors in $S$, and so it has at most one neighbor in $S$.

As a result, $e(G[U]) \leq e(H)-\Delta-(\Delta-1)+1+1$ and $e(G[U]) \geq e(H)-\Delta$, so $\Delta \leq 3$. If equality occurs then, by Lemma 13, part (1), $H_{3}=\left\{y_{k}\right\}$ and $y_{k} \sim H_{2}$. Moreover, the single member of $N(v) \cap S$ is neither $y_{k}$ nor $y_{k-1}$. Furthermore, equality also implies that it is not possible to find a $y_{k-1}$ adjacent to $v$. Hence, $v \nsim H_{3} \cup H_{2}$.

\subsection{Proof of Lemma 10}

Let $\Delta(H)=1$ and $H \notin\left\{K_{2}, \overline{K_{2}}, \overline{P_{3}}\right\}$. Hence, $k \geq 4$ and $\Delta(H) \leq k-3$. Let $G \longrightarrow H$, where $G$ is a graph on $n \geq k+2$ vertices. By Lemma $1, \Delta(G)=1$. We have the following cases:

CASE 1. $G$ has more isolated vertices than $H$.

Color the isolated vertices of $G$ with as many colors as possible using at most $k-1$ colors, color the vertices of degree 1 in $G$ with the remaining colors. It is clear that any rainbow subgraph on $k$ vertices will have more isolated vertices than $H$.

CASE 2. $G$ has more edges than $H$ and $H$ has at least three isolated vertices.

In this case, the number of vertices of degree 1 in $H$ is at most $k-3$. Color vertices in as many edges of $G$ as possible with distinct colors, using at most $k-1$ colors, and color 
the rest of the graph with the remaining colors. Any rainbow subgraph on $k$ vertices will contain more edges than $H$.

CASE 3. $G$ has more edges than $H$ and $H$ has at most two isolated vertices.

Assume first that $n \geq k+3$, then color each of the isolated vertices of $G$ with distinct colors and, for as many edges as possible, color the endvertices with the same color, a different color on each edge. We see that any rainbow $k$-vertex subgraph has at least 3 isolated vertices.

Assume now that $n=k+2$. If $G$ has at least one isolated vertex, then color the endpoints of one edge with color 1, color the endpoints of another edge with color 2, rainbow color the rest. Then, $H$ must have three isolated vertices, a contradiction. Thus, $G$ is a matching. If, as before, we color the vertices of one edge with color 1 , then the vertices in another edge with color 2 and the rest with remaining colors, then $H \approx M_{k / 2+1}^{\prime}$.

To complete the proof, observe that any coloring of $G \approx M_{k / 2+1}$ with $k$ colors gives such a rainbow $H \approx M_{k / 2+1}^{\prime}$.

\subsection{Proof of Lemma 11}

Let $\Delta=2, H \notin\left\{\Lambda, \overline{S_{4}}\right\}$.

CASE 1. $H$ has two nonadjacent vertices of degree 2.

If $k \leq 4$, the only possibility is $H \approx C_{4}$. Corollary 2 from the paper [2] gives that $f(H)=k$ for any regular graph $H$.

Hence, we may assume $k \geq 5$ and $\Delta=2 \leq k-3$. By Lemma 1 part (1), $\Delta(G)=\Delta=2$. We shall show that $G$ is a disjoint union of cycles each of length at least 5 .

Claim 1. Let $S \subset V(G)$ be any vertex set that induces a copy of $H$. Then $V(G) \backslash S$ is an independent set.

For all $v \in V(G) \backslash S$ Lemma 13 part (1) gives that $\left|N(v) \cap\left(S \backslash\left\{y_{k}, y_{k-1}\right\}\right)\right|=\Delta=2$. Each vertex in $V(G) \backslash S$ sends 2 edges into $S$, and thus no edges into $V(G) \backslash S$. So $V(G) \backslash S$ induces an empty graph.

Claim 2. $G$ is a union of cycles.

Assume that there is a vertex $v$ in $G$ such that $\operatorname{deg}(G, v)<2$, consider any two adjacent vertices $w, w^{\prime}$. Color $v, w, w^{\prime}$ with one color and the rest of the graph with the remaining $k-1$ colors. Let $U \subseteq V(G)$ induce a rainbow copy of $H$ under this coloring. Then $U$ contains one vertex from $\left\{v, w, w^{\prime}\right\}$, moreover $v \in U$, since its degree is less than 2. On the other hand, both $w$ and $w^{\prime}$ cannot be in $V(G) \backslash U$ since they are adjacent, 
contradicting the fact that $V(G) \backslash U$ is an independent set. Thus there is no vertex of degree less than 2 in $G$. Therefore $G$ is 2-regular, and Claim 2 follows.

Claim 3. $G$ has no $K_{3}$ and no $C_{4}$.

Assume that $G$ has a triangle. Color its three vertices with the same color and color the rest of the vertices with new colors. In this coloring, there is a pair of adjacent vertices outside of a rainbow copy of $H$, a contradiction. If $G$ has a $C_{4}$, then color each of the two nonadjacent vertices of a copy of $C_{4}$ with color 1 , color each of the two other vertices of $C_{4}$ with color 2 and the color the rest of the vertices of $G$ with the remaining $k-2$ colors (here $n \geq k+2$ is necessary). This gives two adjacent vertices outside of a rainbow copy of $H$, a contradiction.

If $n \geq k+3$, then color four consecutive vertices on one cycle with one color, and color the rest of the vertices arbitrarily with the remaining $k-1$ colors. As a result, there is an edge outside of the rainbow copy of $H$, a contradiction.

Thus we may assume that $n=k+2$. Color three consecutive vertices on one of the cycles of $G$ with color 1 and rainbow color the rest of the vertices with the remaining colors. Since there is no edge outside of a copy of $H$ in $G$, we must pick the middle vertex of color 1 in a rainbow copy of $H$. Thus, $H$ is a disjoint union of an isolated vertex, a path and perhaps some cycles. If $G$ has at least two cycles, then color two vertices in one cycle with color 1, color two vertices in another cycle with color 2 and rainbow color the rest of the vertices with the remaining $k-2$ colors. Under this coloring, $H$ has no isolated vertex, a contradiction. The only case that remains is that $G$ is a single cycle and $H=P_{k-1}+K_{1}$. Since $H$ has two nonadjacent vertices of degree two, $k-1 \geq 5$, thus $n \geq 8$. Let $v, v^{\prime}$ and $u, u^{\prime}$ be two pairs of consecutive vertices of $G$ such that $G-u-u^{\prime}-v-v^{\prime}$ consists of two paths, each of length at least 2. Color $v, v^{\prime}$ with color 1 and $u, u^{\prime}$ with color 2 , rainbow color the rest of the vertices with remaining colors. Under this coloring, $H$ has no isolated vertices, a contradiction.

CASE 2. All degree 2 vertices of $H$ are adjacent.

Recall that $n \geq k+2$. We have that $H$ has one component $L \in\left\{P_{3}, K_{3}, P_{4}\right\}$, and all other components are isolated edges and vertices. We may assume that $k \geq 4$ since $H \notin \mathcal{F}_{\infty}$. If $k=4$ then $H \in\left\{P_{4}, \Lambda, \overline{S_{4}}\right\}$. Since $H \notin \mathcal{F}_{\infty}$, we see that $H \approx P_{4}$. Since $n \geq k+2=6$, Ramsey's theorem gives that $G$ contains either $K_{3}$ or $\overline{K_{3}}$, contradicting the Deck Lemma (Lemma 3). We can assume that $k \geq 5$, giving that $\Delta=2 \leq k-3$ and $\Delta(G)=2$ by Lemma 1 . The Deck Lemma implies that each connected subgraph of $G$ on $|V(L)|$ vertices is isomorphic to $L$.

Assume first that $k \geq 7$. There is only one component of $G$ with at least three vertices (call such a component large) and this large component is either $P_{3}, K_{3}$ or $P_{4}$. Indeed, otherwise one can find two nonadjacent vertices of degree 2 in $G$; considering these and their neighbors will contradict the Deck Lemma. Since $n \geq k+2$, we can color the vertices of the large component of $G$ with two colors and color the remaining vertices arbitrarily. 
Under this coloring, any rainbow subgraph has components with at most two vertices, a contradiction.

Thus, $k=5$ or 6 and $L \in\left\{P_{3}, K_{3}, P_{4}\right\}$.

Let $k \in\{5,6\}$ and $L \in\left\{P_{3}, K_{3}\right\}$. Then, $G$ has no component on more than three vertices, and each large component of $G$ is isomorphic to $L$. If $G$ has only one component isomorphic to $L$, then color it with two colors, and color the rest arbitrarily, resulting in contradiction. If $G$ has at least two such components, color the vertices in two copies of $L$ with two colors each and color the rest of $V(G)$ with the remaining $1 \leq k-4 \leq 2$ colors. Under this coloring, no rainbow subgraph has a component with more than 2 vertices.

Let $k=6$ and $L \approx P_{4}$. Then, $H$ is either $P_{4}+K_{2}$ or $P_{4}+2 K_{1}$. Any component of $G$ on at least three vertices must be $P_{4}$ since otherwise there is a subgraph $P_{5}$ of $G$ which contains two vertices of degree 2 , nonadjacent in $G$, such that these two vertices and their neighbors span at most 5 vertices, which contradicts the Deck Lemma. Color the vertices of $P_{4}$ in $G$ with three colors and color the rest of the graph with the remaining three colors. Under this coloring, no rainbow subgraph has a $P_{4}$.

Let $k=5$ and $L \approx P_{4}$. Then $H \approx P_{4}+K_{1}$. In particular, we have that $H$ has no induced $2 K_{2}$. Thus, $G$ has only one nontrivial (with at least one edge) component and this component is either $P_{4}$ or $C_{5}$. Since $n \geq k+2$, we can color this component with three colors and color the rest of the vertices arbitrarily with the remaining 2 colors, arriving at a contradiction.

\subsection{Proof of Lemma 12}

Recall that $\Delta=\Delta(H)=3, \delta=\delta(H) \leq 1$ and $\delta(G)<n-k+\delta$.

Claim 1. There exists a set $S \subset V(G)$ and vertices $y_{1} \in S$ and $v_{1} \in V(G) \backslash S$ such that $G[S] \approx H, y_{1}$ is a minimum-degree vertex in $G[S]$ and $N\left(v_{1}\right) \cap S=\left\{y_{1}\right\}$.

If $\delta=1$, then this follows directly from Lemma 9 .

If $\delta=0$, then suppose the claim is false. Lemma 13, part (3) gives that there is a $v_{0} \in V(G) \backslash S$ such that $S_{0}:=\left\{v_{0}\right\} \cup\left(S \backslash\left\{y_{k}\right\}\right)$ and $G\left[S_{0}\right] \approx H$. In this case, $\left|N\left(v_{0}\right) \cap\left(S \backslash\left\{y_{1}, y_{2}\right\}\right)\right| \leq \delta+1=1$, so $v_{0} \sim\left\{y_{1}, y_{2}\right\}$ and Lemma 13, part (2) also gives that $y_{1}$ is the unique isolated vertex in $G[S]$. We have the freedom to choose $y_{2}$ to be any degree-one vertex in $G[S]$, hence $v_{0}$ is adjacent to every vertex of degree at most one in $G[S]$.

As a result, $G\left[S_{0}\right]$ has no isolated vertices, a contradiction to the claim that $G\left[S_{0}\right] \approx H$. This proves Claim 1.

Claim 2. $\left|H_{3}\right|=1, H_{3} \sim H_{2}$.

If $\delta=1$, this follows directly from Lemma 9. If $\delta=0$, this comes from Claim 1 and Lemma 13, part (1). This proves Claim 2. 
CASE 1. $\delta=0$.

By Lemma 13, part (2), the graph $G[S]$ has a unique isolated vertex, $y_{1}$. Since $\left|H_{3}\right|=1$, the component containing $y_{k}$ has an odd number of degree-one vertices.

If the component of $G[S]$ containing $y_{k}$ has 3 degree-one vertices, call two of them $y_{2}$ and $y_{3}$. Let $S^{\prime}=\left\{v_{1}\right\} \cup\left(S \backslash\left\{y_{2}, y_{3}\right\}\right)$, where $v_{1}$ as in Claim 1. The graph $G\left[S^{\prime}\right]$ has no isolated vertices. Since $\left|S^{\prime}\right|=k-1$ and $e\left(S^{\prime}\right)=e(H)-1$, the Deck Lemma implies that $S^{\prime}$ is obtained by deleting a degree-one vertex from $H$, which would yield at least one isolated vertex, a contradiction.

Therefore, we may assume that the component of $G[S]$ containing $y_{k}$ has exactly 1 degree-one vertex. Since all degree-two vertices must be adjacent to $y_{k}$, the vertex set $\left\{y_{k}, y_{k-1}, y_{k-2}\right\}$, for two degree-two vertices $y_{k-1}$ and $y_{k-2}$, induces a triangle. Let $S^{\prime \prime}=\left\{v_{1}\right\} \cup\left(S \backslash\left\{y_{k-1}, y_{k-2}\right\}\right)$. The graph $G\left[S^{\prime \prime}\right]$ has no isolated vertices. Since $\left|S^{\prime \prime}\right|=k-1$ and $e\left(S^{\prime \prime}\right)=e(H)-2$, the Deck Lemma implies that $S^{\prime \prime}$ is obtained by deleting a degreetwo vertex from $H$, which would yield at least one isolated vertex, a contradiction.

Hence, there is no graph in CASE 1.

CASE 2. $\delta=1$.

If $y_{1} \not y_{k}$, then let $S^{\prime}=\left\{v_{1}\right\} \cup\left(S \backslash\left\{y_{k}, y_{1}\right\}\right)$, where $v_{1}$ as in Claim 1. Since $\left|S^{\prime}\right|=k-1$ and $e\left(S^{\prime}\right)=e(H)-4$, the Deck Lemma is contradicted. Therefore, we may assume that $y_{1} \sim y_{k}$.

If $H$ is not connected, then since every degree-two vertex in $G[S]$ is adjacent to $y_{k}$, every connected component of $G[S]$ not containing $y_{k}$ must be an isolated edge. Let $\left\{y_{2}, y_{3}\right\}$ be a component of $G[S]$ not containing $y_{k}$. If $S^{\prime \prime}=\left\{v_{1}\right\} \cup\left(S \backslash\left\{y_{2}, y_{3}\right\}\right)$, then $e\left(S^{\prime \prime}\right)=e(H)$, contradicting the Deck Lemma. Therefore, we may also assume that $H$ is connected.

Since $H_{3} \sim H_{2}, y_{1} \sim y_{k}$ and $H$ is connected, there are only three possibilities for $H$ : one for each of $k=4,5,6$. If $k \leq 4$, then $H \approx \bar{\Lambda}$. If $k \in\{5,6\}$ then Figure 3 gives these graphs and the possible ways for $v_{1}$ to be adjacent to $S$.
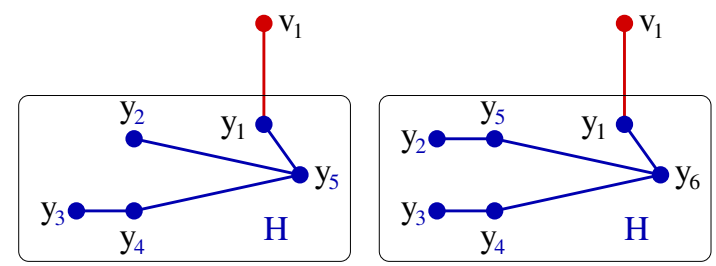

Figure 3: Small graphs, $\delta=1, \Delta=3$ in Lemma 12

In the case where $k=5$, the vertex set $\left\{v_{1}, y_{1}, y_{3}, y_{4}\right\}$ induces the graph $2 K_{2}$, which is not in $\operatorname{deck}(H)$. In the case where $k=6$, the vertex set $\left\{v_{1}, y_{1}, y_{2}, y_{3}, y_{6}\right\}$ induces the graph $P_{3}+2 K_{1}$, which again is not in $\operatorname{deck}(H)$.

Hence, the only graph in CASE 2 is $H \approx \bar{\Lambda}$, which must be excluded because $\bar{\Lambda} \in \mathcal{F}_{\infty}$. 


\section{Concluding remarks}

Open question: $H \approx \Lambda, H \approx \bar{\Lambda}$

It is still an open problem to determine $\mathcal{A}$ rrow $(\Lambda)$ and $\mathcal{A}$ rrow $(\bar{\Lambda})$. We see by Lemma 6 that $C_{4}+K_{1} \longrightarrow \Lambda$; it is shown in [2], that for each $t \geq 1$ there is a graph $G$ on $7 t$ vertices such that $G \longrightarrow \Lambda$. A case analysis, which we neglect to include in this paper, gives that for any $G$ of order $6, G \nrightarrow \Lambda$.

Thus, even the weaker problem of determining $\{n:|V(G)|=n$ and $G \longrightarrow \Lambda\}$ is still open.

\section{Generalizing the problem}

A natural generalization of this problem is as follows: Let $\mathcal{H}$ be a set of $k$-vertex graphs and define $G \longrightarrow \mathcal{H}$ so that if $V(G)$ is colored with $k$ colors, then there is an $H \in \mathcal{H}$ such that $G$ contains a rainbow induced subgraph isomorphic to $H$. Determine $\mathcal{A} \operatorname{rrow}(\mathcal{H})=\{G: G \longrightarrow \mathcal{H}\}$, for interesting sets $\mathcal{H}$ of graphs.

\section{Acknowledgements}

We thank an anonymous referee for helpful comments. We also profoundly acknowledge and thank Chris Godsil for helpful remarks and aiding with the proof of Lemma 7.

\section{References}

[1] J. Akiyama, G. Exoo, and F. Harary, The graphs with all induced subgraphs isomorphic, Bull. Malaysian Math. Soc. (2) 2 (1979), no. 1, 43-44.

[2] M. Axenovich, On subgraphs induced by transversals in vertex-partitions of graphs. Electron. J. Combin., 13(1) (2006), R36.

[3] M. Axenovich and J. Balogh, Graphs having small number of sizes on induced $k$ subgraphs, to appear.

[4] M. Borowiecka-Olszewska, E. Drgas-Burchardt and P. Mihók, Minimal vertex Ramsey graphs and minimal forbidden subgraphs. Discrete Math. 286 (2004), no. 1-2, 31-36.

[5] A.E. Brouwer, A.M. Cohen and A. Neumaier, Distance Regular graphs. Ergebnisse der Mathematik und ihrer Grenzgebiete (3) [Results in Mathematics and Related Areas (3)], 18. Springer-Verlag, Berlin, 1989. xviii+495 pp.

[6] J. Brown and V. Rödl, A Ramsey type problem concerning vertex colorings, J. Combin. Theory Ser. B 52 (1991), no 1., 45-52.

[7] J. Bosák, Induced subgraphs. Finite and infinite sets, Vol. I, II (Eger, 1981), 109118, Colloq. Math. Soc. János Bolyai, 37, North-Holland, Amsterdam, 1984. 
[8] P. Cameron, Permutation Groups. London Mathematical Society Student Texts, 45. Cambridge University Press, Cambridge, 1999. x+220 pp.

[9] C. Godsil and G. Royle, Algebraic Graph theory. Graduate Texts in Mathematics, 207 Springer-Verlag, New York, 2001. xx+439 pp.

[10] C. Godsil, private communication.

[11] P. Hafner, On the graphs of Hoffman-Singleton and Higman-Sims. Electron. J. Combin. 11 (2004), no. 1, Research Paper 77, 33 pp. (electronic).

[12] A.J. Hoffman and R.R. Singleton, On Moore graphs with diameters 2 and 3. IBM J. Rs. Develop. 4 (1960), 497-504.

[13] W.A. Deuber, Canonization. Combinatorics, Paul Erdös is eighty, Vol. 1, 107-123, Bolyai Soc. Math. Stud., János Bolyai Math. Soc., Budapest, 1993.

[14] N. Eaton and V. Rödl, A canonical Ramsey theorem. Random Structures Algorithms 3 (1992), no. 4, 427-444.

[15] R. Graham, B. Rothschild and J. Spencer, Ramsey theory. Second edition. WileyInterscience Series in Discrete Mathematics and Optimization, New York, 1990. xii+196 pp.

[16] T. Łuczak, A. Ruciński and S. Urbanski, Vertex Ramsey properties of families of graphs. J. Combin. Theory Ser. B 84 (2002), no. 2, 240-248.

[17] E.W. Weisstein, "Petersen Graph." From MathWorld-A Wolfram Web Resource. http://mathworld.wolfram.com/PetersenGraph.html

[18] E.W. Weisstein, "Hoffman-Singleton Graph." From MathWorld-A Wolfram Web Resource. http://mathworld.wolfram. com/Hoffman-SingletonGraph.html

[19] D. West, Introduction to Graph Theory, Second Edition, Prentice Hall (2001), xx+588 pp. 